\title{
Fidelity in surgical simulation: further lessons from the S.T.A.R.T.T. course
}

\author{
Garrett G.R.J. Johnson, MD \\ Peter G. Brindley, MD \\ Lawrence M. Gillman, MD, \\ MMedEd
}

Accepted Aug. 14, 2019

\author{
Correspondence to: \\ L.M. Gillman \\ Department of Surgery \\ University of Manitoba \\ GF439 - 820 Sherbrook St \\ Winnipeg MB R3A 1R9 \\ lawrence.gillman@umanitoba.ca
}

DOI: $10.1503 /$ cjs. 017818

\section{SUMMARY}

Simulation has become a popular and ubiquitous medical education tool. In response to learner demands, and because of technological advancement, there is a trend toward increasing the realism of simulation. However, there is a paucity of evidence regarding what degree of fidelity is needed to deliver optimal simulation-based medical education. Feedback from the Simulated Trauma And Resuscitation Team Training (S.T.A.R.T.T.) course suggests that higherfidelity simulation is viewed as highly valuable to learners. Research is needed in order to guide the growing demand for higher-fidelity simulation in our medical training curricula and in order to justify or mitigate the associated costs and logistical challenges. imulation-based methodology is quickly becoming integral to surgical and trauma education. ${ }^{1,2}$ It allows individuals and teams to make mistakes without adverse effects and for "repeat-until-right" rehearsal in areas of danger, rarity or weakness. The growth of simulation has also been catalyzed by work-hour restrictions and an increased focus on patient safety. In short, without simulation there are fewer opportunities to acquire, hone and maintain life-saving and limb-sustaining skills without putting patients or practitioners at risk. ${ }^{2}$ Fortunately, there is mounting evidence that simulation is effective for the development, maintenance and evaluation of both technical and nontechnical clinical skills. ${ }^{1}$ Studies also suggest that supplementary simulation-based practice is superior to traditional, service-based education, which relies on random patient encounters. ${ }^{2}$ It would follow that higherfidelity simulation would do this job better; however, for a variety of factors, this may not be the case.

The term "fidelity" is used to describe how closely a simulation approximates reality; however, the term is not standardized in medical literature. ${ }^{2}$ Typically, "low fidelity" refers to less complex or more artificial simulations, and "high fidelity" to more complex and more realistic simulations. ${ }^{1}$ However, this is an over-simplification, and other components of fidelity have been proposed. ${ }^{1,2}$

Physical or engineering fidelity refers to how close to reality a simulator is in physical or visual appearance. This includes how a replica looks, feels and interacts with the learner, and the environment (location, backdrop, sights, smells) in which the simulation takes place. Psychological fidelity refers to the extent that the skills of the real task are captured by the simulation activity. ${ }^{1}$ Fidelity can be further described by its conceptual fidelity; namely, the sequence of events in the simulation environment and whether they are believable to the learner. For example, if a patient is hemorrhaging, a high conceptual fidelity simulation will have the mannequin also become hypotensive and tachycardic. Experiential or emotional fidelity refers to the learner's thoughts, feelings and emotions toward the simulation; namely, whether the learner "buys in." Together, all of these aspects of fidelity determine the realism of a simulation. ${ }^{2}$ 
The Simulated Trauma and Resuscitation Team Training (S.T.A.R.T.T.) ${ }^{3}$ course provides high-fidelity simulation to multidisciplinary trauma teams with a focus on crisis resource management (CRM) skill development. The course combines physicians, surgeons, registered nurses (RNs), respiratory therapists (RTs) and prehospital personnel in order to practise communication, teamwork, leadership, followership, situational awareness and resource use. The contents of the course and methodology have been described previously. ${ }^{3}$ Recently, in order to further increase course fidelity, we have incorporated standardized patients (SPs) wearing trauma cut-suits, otherwise known as "Human Worn Partial Task Surgical Simulators" (Strategic Operations, Inc.). Standardized patients wear these onepiece zip-up suits in order to mimic traumatic injuries, while participants perform life-saving procedures in real time and on a live person who can react (but is protected). This addition has been well received by course participants over the past 4 courses ( $n=92$; Fig. 1 ), with more than $70 \%$ of participants reporting the suits as an essential part of the course and more than $90 \%$ reporting the suits as valuable to the course and an improvement in course realism. ${ }^{3}$

Despite the putative importance of fidelity, there are few studies comparing high-fidelity simulations with lower-fidelity alternatives. The few that do exist suggest that the level of required fidelity depends on the learning objectives. These studies appear to have focused on educational impact rather than important psychometric factors such as participants' attitudes, feelings and emotional stress response. ${ }^{1,2}$ In addition, the "ideal" level of fidelity for learning a given skill is not known. For example, ever since the 1908 Yerkes-Dodson law there has been an assump- tion that optimal performance requires a degree of physiologic and mental arousal alongside an acceptance that excessive stimulation can overwhelm a learner's cognitive bandwidth. Regardless, there is a tendency to pursue ever higher physical fidelity, even at the expense of functional utility. ${ }^{1,4}$ This phenomenon is referred to as "naïve realism" ${ }^{4}$ and makes it difficult to measure the value of fidelity in simulation.

Higher-fidelity simulation has intuitive potential benefits. For example, when addressing complex skills, such as patient communication, or team dynamics during stress, higher fidelity means including subtle but important intricacies, such as the feel of a tense, crowded resuscitation bay, that may otherwise be difficult to replicate outside of a highly realistic setting. It is also believed that individuals with a higher level of training/expertise in certain procedural skills require higher-fidelity simulations in order to accrue benefit. It is theorized that with higher levels of expertise, a lower-fidelity model could lead to negative transfer; ${ }^{1}$ namely, that previous knowledge affects the ability to learn a new task. Regardless, higher-fidelity simulations may be more enjoyable to the learner, may be regarded as more challenging and useful, and thus increase participation and immersion.

High-fidelity simulation typically adds cost, time and logistics. Accordingly, it is less practical for large numbers of naïve learners. ${ }^{1,2}$ Studies have yet to show that increased fidelity leads to improved acquisition or retention, ${ }^{1}$ and even if it does, increasing fidelity beyond a certain threshold may be associated with diminishing returns. For example, in novices learning laparoscopic surgery, a lowerfidelity training box had much better learning outcomes

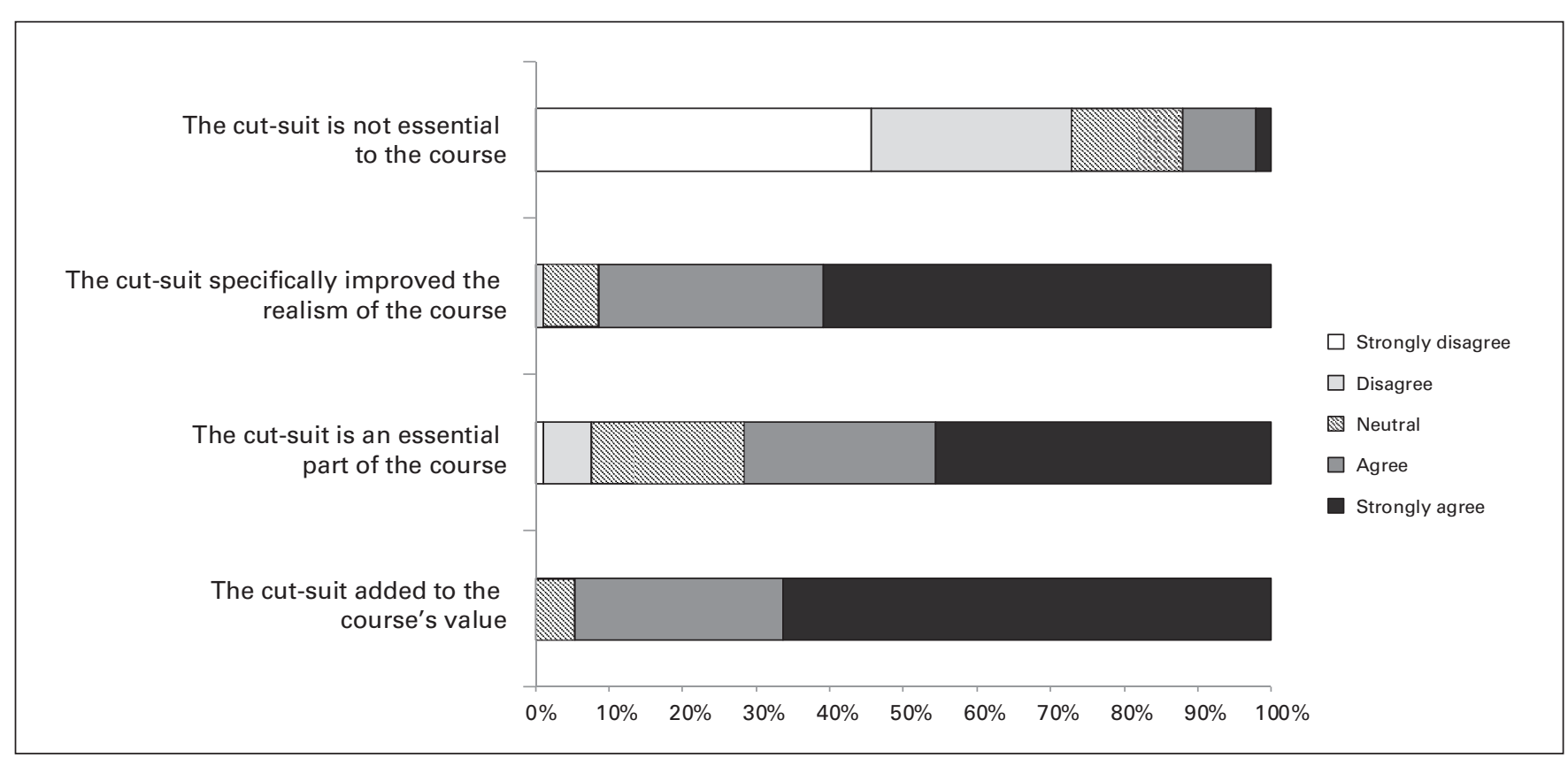

Fig. 1. Participant response to specific cut-suit-related questions on postcourse satisfaction surveys from the last 4 courses since use of the cut-suit began $(n=92)$. 
with regards to skill acquisition than a higher-fidelity virtual reality trainer. ${ }^{5}$ This suggests that beginners learning and practising a skill may not require as much realism.

There are myriad potential benefits to increasing fidelity in simulation, not least of which is that people appreciate it and believe it is necessary (as in our S.T.A.R.T.T. course). However, the costs can be considerable, and the benefits over low-fidelity alternatives are currently not robustly supported by the available literature. The trend in education appears toward higher-fidelity simulation, but as anything in medicine, we should strive to be evidence based and financially prudent. Research is therefore an appropriate investment. With regards to the S.T.A.R.T.T. course specifically, future steps should include evaluating courses with and without the cut-suits to investigate if there is any objective incremental benefit. The real challenge becomes defining what is a true benefit and how it can be objectively measured. Ultimately, we need to harness the opportunities proffered by simulation, and this starts by knowing when the costs are justified. It may be that sometimes less is more and, ultimately, tailoring the fidelity of the simulation to the learners' needs remains the key to making cost- and resource-conscious but still effective simulations.
Affiliations: From the Department of Surgery, Section of General Surgery, University of Manitoba, Winnipeg, Man. (Johnson, Gillman); and the Department of Critical Care Medicine, Anesthesiology, University of Alberta, Edmonton, Alta. (Brindley).

Competing interests: None declared.

Contributors: All authors contributed substantially to the conception, writing and revision of this article and approved the final version for publication. No other competing were declared.

\section{References}

1. Munshi F, Lababidi H, Alyousef S. Low- versus high-fidelity simulations in teaching and assessing clinical skills. 7 Taibah Univ Med Sci 2015;10:12-5.

2. Nataraja RM, Webb N, Lopez PJ. Simulation in paediatric urology and surgery. Part 1: An overview of educational theory. 7 Pediatr Urol 2018;14:120-4.

3. Gillman LM, Martin D, Engels PT, et al. S.T.A.R.T.T. plus: Addition of prehospital personnel to a national multidisciplinary crisis resource management trauma team training course. Can f Surg 2016;59:9-11.

4. Smallman HS, St. John M. Naive realism: Misplaced faith in realistic displays. Ergon Des 2005;13:6-13.

5. Steigerwald SN, Park J, Hardy KM, et al. Does laparoscopic simulation predict intraoperative performance? A comparison between the Fundamentals of Laparoscopic Surgery and LapVR evaluation metrics. Am 7 Surg 2015;209:34-9. 